DEPÓSITO LEGAL ZU2020000153

Esta publicación científica en formato digital

es continuidad de la revista impresa

ISSN 0041-8811

E-ISSN 2665-0428

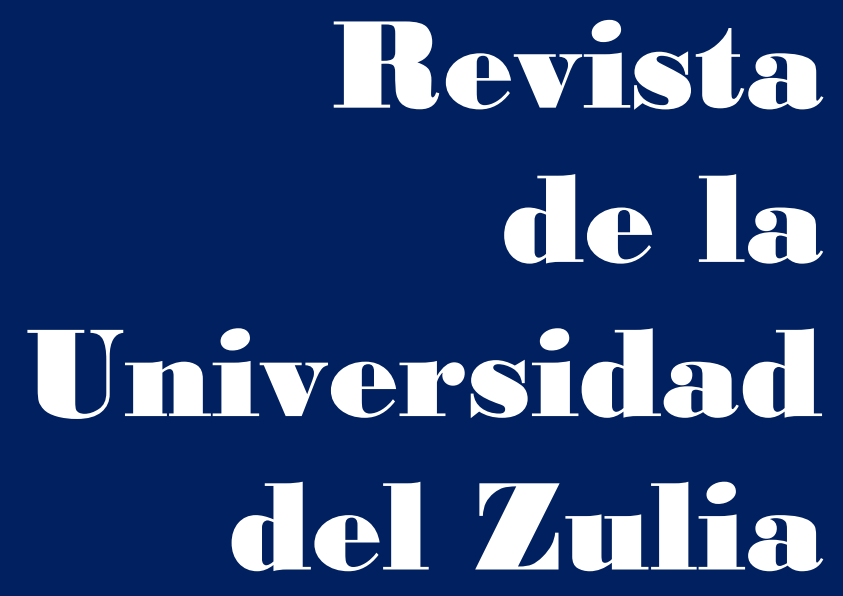

Fundada en 1947

por el Dr. Jesús Emrique Lossada

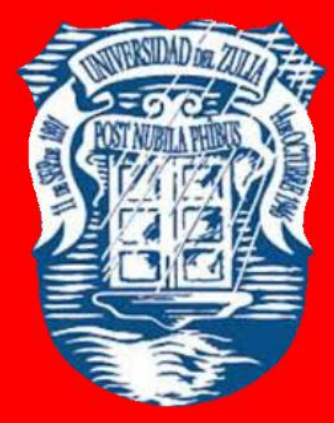

Ciencias

Sociales

y Arte

Año $12 \quad N^{\circ} 34$

Septiembre - Diciembre 2021

Tercera Época

Maracaibo-Veneruela 


\title{
Liderazgo resonante según el género: un estudio en las pequeñas y medianas empresas de la ciudad de Barranquilla
}

\author{
Ricardo Romario Antequera Amaris * \\ Reynier Israel Ramírez Molina ** \\ Mauricio Junior Santamaria Ruiz *** \\ Nelson David Lay Raby ****
}

\begin{abstract}
RESUMEN
La actual investigación identifica al liderazgo resonante según el género en las PYMES panificadoras de la ciudad de Barranquilla - Colombia. Empleando la metodología cuantitativa, de carácter descriptiva, diseño de campo, transversal, y no experimental, figurando como objeto de estudio los setecientas sesenta y nueve (769) miembros colaboradores del sector panificador de la ciudad Barranquilla. Utilizando como técnica, la encuesta y desarrollando el cuestionario como instrumento, éste cuenta con treinta (6) ítems, validado por el juicio de diez (10) expertos, resultando una confiabilidad de $(0,81)$ en base al coeficiente Alfa de Cronbach, valorándose como altamente confiable. Los resultados evidencian que el liderazgo resonante para el indicador género femenino fue el de mayor ponderación, catalogado como presente, mientras el género masculino figura medianamente presente. Se concluye que lo imperativo y relevante de tener y potencializar competencias que permitan la adaptación según los requerimientos organizacionales y del mercado, fomenta la equidad de género, sin desmeritar factores de la empresa, manteniendo los principios de responsabilidad corporativa, social y ambiental, transformación ante el cambio, todo esto apoyado en la innovación.
\end{abstract}

PALABRAS CLAVE: liderazgo; género; pequeña empresa; mercado.

Este artículo es producto de la investigación titulada "Liderazgo resonante en las PYMES panificadoras de la ciudad de Barranquilla", trabajo de grado del Programa de Administración de Empresas, Departamento de Ciencias Empresariales, articulado con el Semillero de Investigación de Gestión de Talento Humano (SIGTH), y enmarcado en el Proyecto de Aula de las asignaturas Procesos de Talento Humano y Direccionamiento estratégicos, de la Universidad de la Costa, Atlántico - Barranquilla, Colombia.

*Estudiante del programa de administración de empresas y Miembro del Semillero de Investigación de Gestión de Talento Humano (SIGTH) de la Universidad de la Costa, Atlántico - Colombia. Dpto. de Ciencias Empresariales. ORCID: https://orcid.org/0000-0003-3949-0855. Email: ranteque2@cuc.edu.co

**Profesor e Investigador Tiempo Completo de la Universidad de la Costa, Atlántico - Colombia. Dpto. de Ciencias Empresariales. ORCID: https://orcid.org/0000-0002-5073-5158. Email: rramirezl3@cuc.edu.co

*** Estudiante del programa de administración de empresas y Miembro del Semillero de Investigación de Gestión de Talento Humano (SIGTH) de la Universidad de la Costa, Atlántico - Colombia. Dpto. de Ciencias Empresariales. ORCID: https://orcid.org/0000-0002-2976-0834. Email: msantama@cuc.edu.co

****Profesor Full Time, Facultad de Educación y Ciencias Sociales, Universidad Andrés Bello, Viña del Mar Chile. ORCID: https://orcid.org/0000-0001-8501-7570. Email: nelson.lay@unab.cl

Recibido: 14/06/2021

Aceptado: 29/07/2021 


\section{Resonant leadership according to gender: a study in small and medium-sized companies in the city of Barranquilla}

ABSTRACT

The current research identifies the resonant leadership according to gender in the bakery SMEs of the city of Barranquilla - Colombia. Using the quantitative, descriptive, field design, crosssectional, and non-experimental methodology, the seven hundred and sixty-nine (769) collaborating members of the bakery sector of the city of Barranquilla appear as the object of study. Using the survey as a technique and developing the questionnaire as an instrument, it has thirty (6) items, validated by the judgment of ten (10) experts, resulting in a reliability of (0.81) based on Cronbach's Alpha coefficient, valuing itself as highly reliable. The results show that the resonant leadership for the female gender indicator was the one with the highest weight, cataloged as present, while the male gender is moderately present. It is concluded that the imperative and relevant of having and potentiating skills that allow adaptation according to organizational and market requirements, promotes gender equity, without detracting from company factors, maintaining the principles of corporate, social and environmental responsibility, transformation before change, all this supported by innovation.

KEY WORDS: leadership; gender; small enterprises; markets.

Introducción

El logro de una organización podría estar dado por la ejecución y cumplimiento de objetivos, todo esto respaldado por la satisfacción y rendimiento de los colaboradores, por lo que el activo humano se ha establecido como un componente básico para la consecución de metas y objetivos, siendo imperativo contar con personas capaces y excepcionalmente equipadas, que hagan posible el perfeccionamiento de los procesos y métodos. Además, las actuales situaciones de crisis y búsqueda de una recuperación económica exigen nuevos modelos e ideas para progresar mediante la ejecución de los planes estratégicos. Esto requiere colaboradores con competencias que los comprometan a ocuparse de sus recursos humanos y, además, a enfrentar las dificultades del mercado.

Se puede plantear el liderazgo como una de esas habilidades clave; investigadores han conceptualizado esta variable desde varias perspectivas, así mismo su idea ha avanzado a lo largo 
del tiempo y la cultura, presentando la mezcla de diferentes aptitudes, como el cumplimiento de objetivos, mejora individual y grupal, la comunicación, motivación y métodos de retribución (Marañón et al, 2021). El liderazgo se distingue por ser situacional, el cual implica condiciones y circunstancias que se ajustan a los cambios que puedan ocurrir. A nivel mundial, el liderazgo se considera un instrumento eficaz para el direccionamiento de personal y a su vez para hacer frente a las transformaciones que puedan producirse a causa de las crisis, sectores dinámicos y competencia, por lo que el líder ha de vencer dificultades para cumplir objetivos financieros, sociales, ambientales y tecnológicos, descubriendo oportunidades, gestionando al personal y mejorando beneficios para los asociados.

En Colombia han aparecido estilos de liderazgo para hacer frente a las dificultades introducidas por el mercado, independientemente que sean individuales o autoritarios, se reflejan carencias en el desarrollo de las aptitudes de liderazgo. Los estudios sugieren reexaminar los enfoques convencionales de preparación y aprendizaje para otros más productivos, para garantizar un descubrimiento genuino que impulse un cambio individual para llegar a una administración efectiva a nivel humano y autoritario.

Mientras que en el distrito caribeño se percibe un liderazgo situacional adecuadas a las necesidades del entorno y su gente. El liderazgo, como indican Hughes et al. (2007), Fernández et al. (2020) y Sukier et al. (2020), experimenta experiencias que abarcan emociones, acciones y decisiones apoyados en la razón y lógica. De modo que los líderes son miembros que conducen a la obtención de organizaciones competitivas y con alto grado de cumplimiento de los objetivos organizacionales. Robbins (2004), caracteriza el liderazgo como la naturaleza de incidir en las personas para lograr los destinos fijados.

Así, Goleman et al. (2016), conceptualizan el liderazgo como la actividad de los líderes que buscan comprender y empatizar las emociones y situaciones de las personas, para situarlas hacia un rumbo positivo. Es posible que esto ocurra cuando el líder motiva a sus compañeros de equipo para que desarrollen sentimientos y emociones beneficiosas para la empresa, de la misma manera que debe tener la capacidad de relacionarse y extender sus emociones hacia las personas de la empresa. Para quienes investigan, el liderazgo resonante sale del esquema de liderazgo convencional y presenta otro tipo, donde los líderes tienen la capacidad de motivar y preparar a 
sus compañeros hacia un objetivo, esto se origina como resultado de la encauzación emocional por parte del líder, construyendo en ellos seguridad, confianza y respeto, creando un fuerte vínculo para avanzar juntos. Según Ramírez et al., (2019) y Ramírez (2020), el liderazgo resonante se define como la capacidad que posee el talento humano de armonizar y generar emociones positivas, capaz de cautivar a su gente, diseñando estrategias de gestión para acompañar al ser vivo y experimentar sensaciones de bienestar consciente y responsablemente.

De lo anterior, se expresa que el problema radica en la probable ausencia de un liderazgo resonante, que permita estimular al personal y generar experiencias positivas en la gestión de personas en las organizaciones, provocado por una potencial carencia de capacidades que ayuden a desarrollar un liderazgo asertivo que armonice el entorno, dentro de ellas la ausencia de visión, donde se anticipen los factores que inciden en la organización, de esta manera, el líder no tendría la opción de ajustar su liderazgo como lo indica la circunstancia. Por ello, es importante formar, evaluar y desarrollar estilos que produzcan sinergia y empatía en la gente, redes empresariales, y stakeholders, potencializando competencias que favorezcan la transformación del líder - personas - organización, por lo que surge la siguiente interrogante: ¿Cómo sería el liderazgo resonante según el género en las PYMES panificadoras de la ciudad de Barranquilla?

\section{Liderazgo resonante según género: revisión desde la teoría}

A lo largo de los tiempos las organizaciones han comprendido que existen muchas habilidades en los colaboradores para una mayor cercanía al éxito organizacional, entre ellas se encuentra el liderazgo, según Maxwell (2007), Ramírez et al. (2018) y Ramírez et al. (2021), es el poseer habilidades y capacidades que permitan la persuasión de colaboradores con el ánimo de promover mejoras en las actitudes, aptitudes y capacidades para el cumplimiento de manera eficiente de las estrategias organizacionales, mientras que Hughes et al. (2018) y Ramírez et al. (2019), consideran que el liderazgo tiene la capacidad de modificar la perspectiva en la que los colaboradores se auto perciben, apuntando a obtener grandes logros para la organización e individuo. 
En tal sentido, las empresas han incluido dentro de sus requisitos, habilidades que permitan el desarrollo del liderazgo, sin embargo, muchas de estas desconocen los estilos de liderazgo según la situación, habilidades y personalidad propia. Antequera y Ospino (2021) y Ramírez (2020), definen al líder resonante como aquel capacitado para identificar y comprender los sentimientos de sus colaboradores, permitiéndoles promover sentimientos positivos y acciones efectivas. Convergiendo, Smith et al. (2010), afirman que los líderes con menos posibilidad de crear malos ambientes laborales, cansancio e insatisfacción son los estilos de liderazgos positivos o resonantes.

Posterior de haber expuesto qué es el liderazgo resonante, es fundamental separar y diseccionar la forma en que los líderes se comportan según su género, teniendo en cuenta que cada uno de ellos tiene varios características, aptitudes y habilidades, lo que afecta a la forma en que se practica el liderazgo y, además, en la administración de las personas, autoridad, motivación y comunicación (tabla l).

Tabla 1. Liderazgo resonante según género bajo referentes teóricos

\begin{tabular}{cccc}
\hline Masculino & Femenino \\
\hline $\begin{array}{c}\text { Carocca } \\
(2016)\end{array}$ & $\begin{array}{c}\text { Emplea estilos dominantes y } \\
\text { conflictivos, su objetivo es } \\
\text { obtener el éxito particular. }\end{array}$ & $\begin{array}{c}\text { Carocca } \\
(2016)\end{array}$ & $\begin{array}{c}\text { Emplea estilos } \\
\text { participativos y prioriza el } \\
\text { trabajo cooperativo. }\end{array}$ \\
\hline $\begin{array}{c}\text { Eagle et } \\
\text { al. (2003) }\end{array}$ & $\begin{array}{c}\text { Emplea estilos autoritarios, } \\
\text { independiente, eficaz y se } \\
\text { caracteriza por tener rasgos. }\end{array}$ & $\begin{array}{c}\text { Eagle et } \\
\text { al. } \\
(2003)\end{array}$ & $\begin{array}{c}\text { Se caracteriza por tener } \\
\text { rasgos, prioriza el bienestar, } \\
\text { mantiene buenas relaciones } \\
\text { sociales. }\end{array}$ \\
\hline $\begin{array}{c}\text { Fritzy } \\
\text { Van }\end{array}$ & $\begin{array}{c}\text { Posee rasgos que favorecen } \\
\text { estilos de liderazgo } \\
\text { autoritarios, competitivos, }\end{array}$ & $\begin{array}{c}\text { Lanzoni } \\
(2008)\end{array}$ & $\begin{array}{c}\text { Se fundamenta en la } \\
\text { humización, permitiendo } \\
\text { tener relaciones más } \\
\text { astrechas, más capacidad de } \\
\text { motivación. }\end{array}$ \\
\hline $\begin{array}{c}\text { Miranda enfocados al logro. } \\
(2019)\end{array}$ & $\begin{array}{c}\text { Prioriza estilos que faciliten } \\
\text { la orientación del personal a } \\
\text { desarrollar sus funciones y } \\
\text { tareas. }\end{array}$ & $\begin{array}{c}\text { Brzovic } \\
(2010)\end{array}$ & $\begin{array}{c}\text { Emplea estilos que permiten } \\
\text { desarrollar la empatía, } \\
\text { prioriza el bienestar para } \\
\text { obtener mejoras en el } \\
\text { rendimiento. }\end{array}$ \\
\hline
\end{tabular}

Fuente: elaboración propia (2021). 


\section{Metodología}

\subsection{Paradigma de la investigación}

Es un conjunto de teorías, técnicas y procesos que constituyen una agrupación científica que tengan como finalidad el identificar problemas para de esta manera darle una solución (Ruiz, 1992). Por tanto, el desarrollo investigativo está soportado por uno o más paradigmas, permitiéndole diversas perspectivas sobre cómo analizar y estudiar los objetos y procedimientos (Herrera et al. 2018). Por lo que la investigación que fundamenta el presente artículo se basó en el paradigma positivista acompañado de un método cuantitativo, el cual consistió en la obtención y procesamiento numérico de los datos. Hernández et al. (2014) y Guerrero et al. (2018), sostienen que el conocimiento debe de ser imparcial, ocasionado por un proceso deductivo en base a una medición numérica y un análisis estadístico inferencial, donde se verifican o desvirtúan las hipótesis fijadas.

\subsection{Tipo de investigación}

El actual artículo se enfocó en el liderazgo resonante según género en las PYMES panificadoras en la ciudad de Barranquilla. De tipo descriptiva, a través del análisis de la variable por medio de la compilación de datos desde el origen primario, cuyo objetivo fue describir la situación o problema. Antequera y Ospino (2020), sostienen que la finalidad de la investigación descriptiva es obtener información sobre las variables, creencias y teorías que están vinculadas al objeto de estudio, Dankhe (1986), considera que el estudio descriptivo busca exponer las características o rasgos de una población, individuo o fenómeno que pueda ser estudiado. De la misma manera Malhotra (1997), afirma que la investigación descriptiva tiene como finalidad el conocer y exponer los rasgos y características sobre una situación o problema. Así mismo, Hernández et al. (2014), consideran que el diseño de investigación es transversal, donde la recopilación de datos es determinada y ejecutada en un período único. Además, el objetivo de la investigación transversal es plantar la variable y cuál es su incidencia en un momento definido., facilitando su aplicación a individuos, procesos y demás. Los diseños transeccionales se dividen en: (1) exploratorio, (2) descriptivos y (3) correccionales-causa. 


\subsection{Diseño de la investigación}

Se conceptualiza como un conjunto de métodos, técnicas e instrumentos seleccionados adecuadamente por los investigadores según el fenómeno o el problema a estudiar, el diseño indica de qué manera se llevará la investigación, detallando el instrumento para la recolección, cómo se medirán y que técnica se aplicará para el análisis de los datos. Referente al objeto de estudio, se perfila dentro del diseño de investigación como de campo no experimental. Para Hernández et al. (2014), una investigación no experimental se da en el momento que no se alteran las variables, visualizando y analizando fenómenos en su entorno genuino o real. Por tal motivo se considera el actual trabajo investigativo como transversal, no experimental y de campo.

De igual manera, un diseño transeccional, de tipo descriptivo, según, Hernández et al. (2014), es aquel análisis donde se exponen diversas perspectivas del estado actual de una variable o más, así mismo su vinculación entre uno o más personas. Siguiendo el diseño, se establece como investigación de campo, obteniendo los datos e información del personal operativo, comercial y administrativo de las PYMES panificadoras de la ciudad de Barranquilla. A su vez se categoriza como transeccional en referencia a Hurtado (2010), en vista que la recolección fue dada en un periodo único, precisamente en el periodo 2020; la finalidad fue analizar el liderazgo resonante para compilar y procesar resultados, obteniendo unas conclusiones que nos llevan a emitir recomendaciones.

\subsection{Población de la investigación}

La población estuvo conformada por las PYMES panificadoras de la ciudad de Barranquilla (Always Fresh S.A.S., La Baguette S.A.S., Panificadora Del Litoral S.A., La Frontera S.A.S., Organización Panadería Nueva York S.A.S., Inversisa S.A.S., Galipan de la Costa y Molinos del Atlántico) (Ramos Ruíz et al., 2013, Sectorial, 2016, Instituto Colombiano de Bienestar Familiar, 2017). Conceptualizando la muestra como un subgrupo respecto a la población total, en la investigación actual se delimitó como las empresas categorizadas como PYMES pertenecientes al sector panificador de la ciudad de Barranquilla. Por lo que se empleó 
el muestreo por conglomerado. Las empresas clasificadas como PYMES en Colombia, según decreto 957 de 5 JUN 2019, donde se define la clasificación empresarial, cuyo criterio es el ingreso obtenido por las actividades, así mismo, se secciona en tres macro sectores como: manufactura, servicios y comercio (tabla 2).

Tabla 2. Clasificación según ingresos (en millones) para las PYMES en Colombia

\begin{tabular}{|c|c|c|c|}
\hline $\begin{array}{c}\text { Sector } \\
\text { Tamaño }\end{array}$ & Manufactura & Servicios & Comercio \\
\hline Pequeña & de 811 a 7.027 & de 1.131 a 4.523 & de 1.535 a 14.781 \\
\hline Mediana & de 7.027 a 59.527 & de 4.523 a 16.558 & de 14.78 a 74.066 \\
\hline
\end{tabular}

Fuente: Ministerio de comercio, industria y turismo. Decreto 957 de 5 JUN (2019).

Aplicando los criterios de inclusión, se emplea como muestra ocho (8) empresas de la ciudad de Barranquilla, categorizadas como pequeñas y medianas empresas del sector de panificador, ubicadas en la ciudad de Barranquilla, su estructura organizacional es similar, con un número de colaboradores significativos. La muestra estuvo conformada por setecientas sesenta y nueve (769) sujetos, seleccionados por el número de personas que cuentan a su cargo, estos se agruparán por áreas como (1) operativa, (2) comercial y (3) administrativo. Desempeñando los siguientes cargos: gerente de producción, jefes de: bodega, ventas, almacén y control de calidad.

\subsection{Técnicas e instrumento de recolección de datos}

La técnica que se empleó para la recopilación de datos fue la encuesta, juntamente con el cuestionario como instrumento, este contiene un listado de preguntas de selección múltiple, figurando treinta (30) ítems, utilizando una escala ordinal, conteniendo alternativas de respuesta, $(\mathrm{S})$ : siempre, $(\mathrm{CS})$ : casi siempre, $(\mathrm{AV})$ : a veces, $(\mathrm{CN})$ : casi nunca, $(\mathrm{N})$ : nunca. Por lo que, Bavaresco (2008), lo configura como una herramienta que recopila información desde la fuente primaria. Del mismo modo, Hernández et al. (2014), aseveran que la escala ordinal es el agrupamiento de ítems, que se exponen juicios o afirmaciones, con los cuales se recopila reacciones del objeto estudiado, conforme codifica cada opción de respuesta, evaluando del cinco 
REVISTA DE LA UNIVERSIDAD DEL ZULIA. 3época. Año 12 N $^{\circ} 34,2021$

Ricardo. R. Antequera Amaris et al. /// Liderazgo resonante según el género... 29-44

DOI: http://dx.doi.org/10.46925//rdluz.34.03

(5) al uno (1) las opciones con proposición positivos y del uno (1) al cinco (5) las opciones con proposición negativos (tabla 3).

Tabla 3. Codificación de las opciones de respuestas

\begin{tabular}{|c|c|}
\hline Opciones positivas de Alternativa & Opciones Negativas Ponderación \\
\hline (5) Siempre. & (1) Nunca. \\
\hline (4) Casi Siempre. & (2) Casi Nunca. \\
\hline (3) A veces. & (3) A veces. \\
\hline (2) Casi Nunca. & (4) Casi Siempre. \\
\hline (1) Nunca. & (5) Siempre. \\
\hline
\end{tabular}

Fuente: Hernández et al (2014).

Tamayo y Tamayo (2007), manifiestan que el cuestionario como instrumento cuenta con atributos o aspectos del hecho estudiado, posibilitando separar problemas que reduzcan la validez y realidad en los datos necesarios del objeto en investigación. Exponiendo el propósito del trabajo en curso, se estableció un baremo para comprender la media aritmética fijando el proceder de los indicadores del liderazgo resonante según el género en las PYMES panificadoras de la ciudad de Barranquilla (tabla 4).

Tabla 4. Baremo de categorización de la media aritmética determinando la conducta de la variable liderazgo resonante según el género

\begin{tabular}{|c|c|}
\hline Rango de puntaje & Categorización \\
\hline $1-1,79$ & No presente \\
\hline $1,80-2,59$ & Poco presente \\
\hline $2,6-3,39$ & Medianamente presente \\
\hline $3,4-4,19$ & Presente \\
\hline $4,2-5$ & Muy presente \\
\hline
\end{tabular}

Fuente: Hernández et al (2014).

Se concibe como valido un instrumento cuando este mide para lo que fue diseñado o planeado, puntualmente, la variable u objeto de estudio. Esta fase destaca por las diversas acciones que allí se han de desarrollar, en ellas la elaboración del cuestionario debe de estar acorde a los objetivos. La validez de la información recopilada está directamente relacionada con el instrumento con su contexto teórico. Hernández et al. (2014), afirma que la validez habla sobre 
la eficacia de un instrumento frente a la variable que está bajo objetivo a medir. Asimismo, el autor antes mencionado sostiene que la validez de un cuestionario como instrumento, está dada por el análisis crítico de los factores. En tal sentido se verificó la validez del instrumento mediante el juicio dado por diez (10) expertos, quienes determinarán qué tan adecuado son los ítems en referencia a los objetivos, dimensiones e indicadores del subconjunto de la muestra.

La confiabilidad de un instrumento hace referencia al grado de precisión del proceso de medición, buscando minimizar fallas, sustentando su coherencia y solidez. Buscando de esta la no incidencia dada por costumbres o halos por parte del investigador, con la finalidad de analizar y gestionar los datos compilados. Magnusson (1978), concibe la confiabilidad como la suma del puntaje real y de error, logrando un puntaje que tenga la capacidad de mostrar la ausencia de errores. Según Chávez (2012), la confiabilidad hace referencia al nivel de congruencia referente a la estudio y medición de una variable, para la actual investigación, se emplea la fórmula de Alfa Cronbach. Por tanto, se aplicaron pilotos a doscientos diez y ocho (218) individuos, con rasgos y características semejantes a la población total la cual está siendo estudiada, obteniendo como resultado una confiabilidad del instrumento de $(0,81)$, pudiendo deducir que el instrumento diseñado es fiable basado en el barómetro de interpretación (tabla 5).

Tabla 5. Escala de valoración del coeficiente de confiabilidad

\begin{tabular}{|c|c|}
\hline Escala & Categoría \\
\hline $0-0,20$ & Muy baja \\
\hline $0,21-0,40$ & Baja \\
\hline $0,41-0,60$ & Moderada \\
\hline $0,61-0,80$ & Alta \\
\hline $0,81-1$ & Muy alta \\
\hline
\end{tabular}

Fuente: Hernández et al. (2014).

\subsection{Técnica de análisis de datos}

Posterior de obtener los datos se procede a realizar el análisis, la codificación y tabulación se hizo con la hoja de cálculo en Microsoft Excel (.xlsx), utilizando más tarde la estadística descriptiva; según Cruz et al. (2014), la estadística descriptiva busca establecer conclusiones 


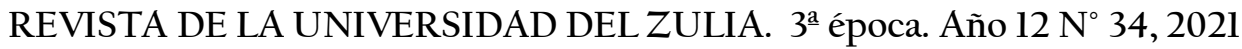

Ricardo. R. Antequera Amaris et al. /// Liderazgo resonante según el género... 29-44

DOI: http://dx.doi.org/10.46925//rdluz.34.03

empleando la inferencia como herramienta, de esta manera obtener perspectivas sobre la población examinada, permitiendo la aprobación de una hipótesis o la representación de la población a través de la medición matemática.

3. Resultados

Tabla 6. Liderazgo resonante según género

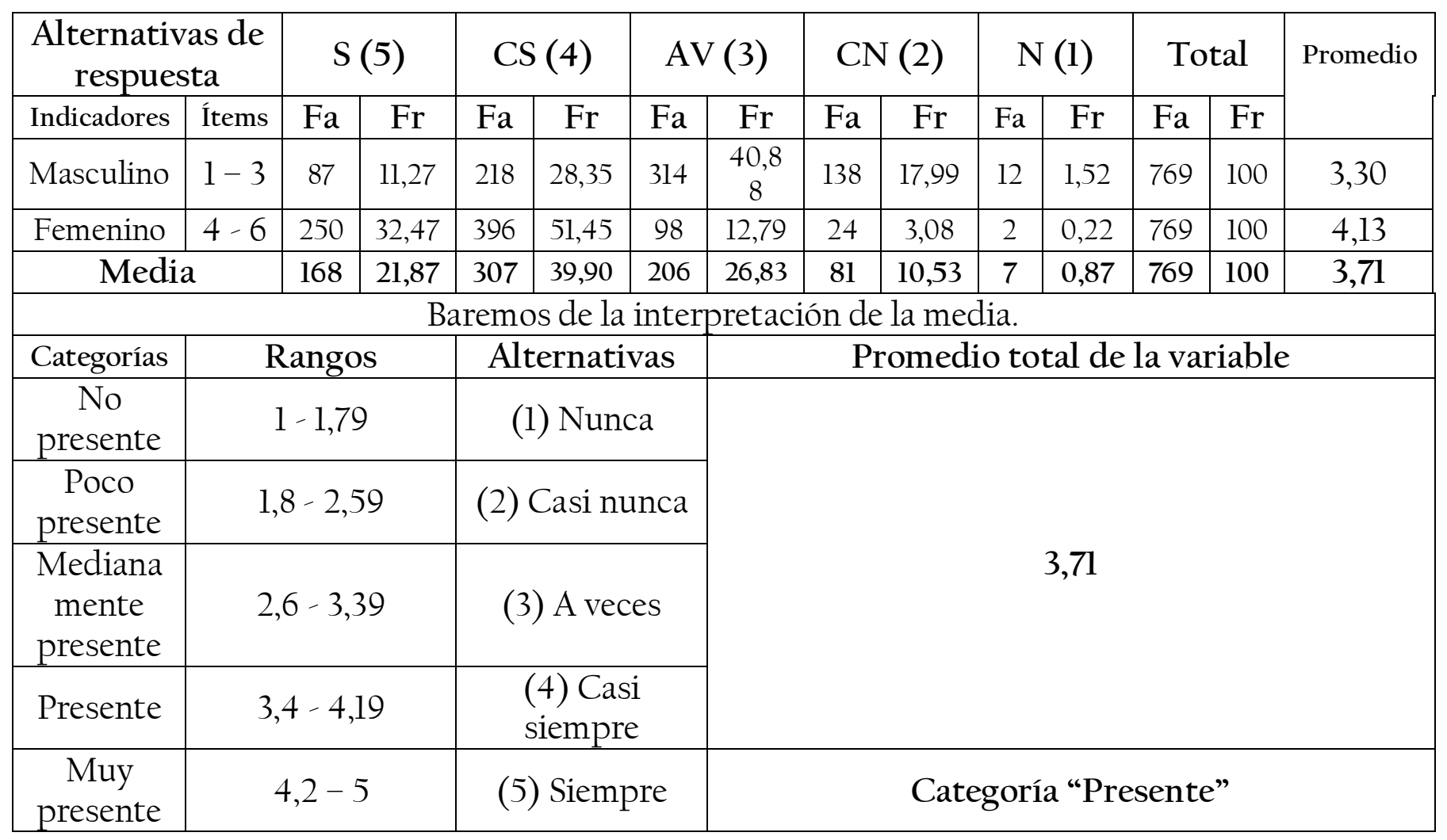

Fuente: elaboración propia (2020).

El liderazgo resonante según el género consta de (2) dos indicadores apoyados cada uno por tres ítems, que se detallan en la tabla 6. Se observa que el indicador "liderazgo resonante según el género masculino" (ítems 1, 2 y 3), obtuvo un promedio relativamente intermedio para esta dimensión, de 3,30 mostrando una tendencia 60,23\%, de respuestas positivas y neutrales respectivamente, con 28,35\%, de opción de respuesta casi siempre y de 40,88\% de opción de respuesta algunas veces. 
Seguidamente se observa el indicador "liderazgo resonante según el género femenino" (ítems 4, 5 y 6); se considera como presente en las Pymes a las que representan los encuestados, debido a un promedio alcanzado de 4,13 fundamentado con un $83,92 \%$ de estos que seleccionaron las opciones siempre y casi siempre (32,47\% y 51,45\% seguidamente, afirmando que este indicador cuenta con una mayor concentración en pertinencia a una tendencia positiva, demostrando de este modo que el género femenino cuenta con características diferenciadoras y estilos orientados la resonancia.

Luego de analizar los resultados obtenidos, la media global hacia el liderazgo resonante según el género resulta altamente positiva, encasillando esta dimensión como presente en las organizaciones que hicieron parte de la muestra del actual estudio. Dentro de esta se evidencia una presencia relativamente alta-moderada, que los líderes de género masculino cuentan con características que se relacionan con el cumplimiento de metas estableciendo un comportamiento adecuado a la asignación y consolidación de tareas, direccionando sus iniciativas a la realización de sus funciones, indiferentemente de su estado de ánimos, circunstancias o incentivos, relacionado con el orden, acatamiento y desempeño laboral.

Mientras que la líder femenina cuenta con rasgos y estilos que buscan un mayor bienestar para su personal, asociado con la empatía, sinergia, armonía y el lenguaje positivo, el desempeño es adecuado, centrado en armonizar su entorno, afectado por factores económicos, ambientales y sociales, que inciden frecuentemente en la capacidad de respuesta de sus labores, y que notoriamente por el tipo de trabajo (operativo), la dinámica de labor disminuye el tiempo para recrear su mente y mantener niveles de productividad altos.

\section{Discusión de resultados}

Detallando la variable liderazgo resonante según su género, un 37,24\%, se obtiene una media global de 3,71, basándose en el baremo de interpretación en referencia al objeto estudiado manifiesta con una tendencia positiva leve que, contempla que los líderes masculinos cuentan con características o rasgos que favorecen el cumplimiento de objetivos, en comparación a las líderes femeninas que cuentan con rasgos diferenciadoras, contrastando con Arteaga y Ramón (2009), exponiendo, la existencia de disparidades entre hombres y mujeres sobre la manera de 
REVISTA DE LA UNIVERSIDAD DEL ZULIA. 3época. Año 12 N $^{\circ} 34,2021$

Ricardo. R. Antequera Amaris et al. /// Liderazgo resonante según el género... 29-44

DOI: http://dx.doi.org/10.46925//rdluz.34.03

ejercer el liderazgo, el hombre suele ser más autoritario y lleva un liderazgo enfocado hacia la tarea, de esta manera alcanzar los objetivos, mientras que las mujer enfatiza en la motivación y en el desarrollo del personal, obteniendo como resultado mayor rendimiento del personal. Buitrago (2014), Ramírez et al. (2019) y Ramírez et al. (2020), consideran que la manera de ejercer el liderazgo por parte de una mujer suele estar relacionada con la estructura más horizontal, en estas se le da suma importancia a la motivación, el trabajo en equipo y relaciones interpersonales, incidiendo en el comportamiento, el clima organizacional y el rendimiento.

El estudio realizado por Ramírez et al. (2020), al valorar el liderazgo resonante según género muestra que fue poco presente, cabe destacar que el indicador masculino, hace referencia a la naturaleza del servicio, mientras que el indicador femenino, casi nunca las mujeres ocupan cargos de mandos de reportes con la habilidad resonante, es decir, que sus características o estilos derivan de acuerdo con el género que posea cada líder en cuestión. Estos resultados, difieren con los actuales resultados de esta investigación, evidenciando que depende del tipo de organización la capacidad de liderazgo resonante según el género sería diferencia, de igual manera que el liderazgo según el género se ve afectado por factores sociales que inciden en su estilo y forma de aplicar en las funciones sustantivas a los cargos.

\section{Conclusiones}

Al revisar la exposición de resultados y su análisis, se concluye que: (1) el liderazgo resonante en las Pymes panificadoras en la ciudad de Barranquilla, muestra un favorecimiento frente a la gestión del personal en relación al género; (2) el género femenino se encuentra más cercano a la resonancia, impactando de manera social y personal a los colaboradores, mientras que el género masculino está medianamente presente y su objetivo va relacionado a la competitividad y consecución de logros; (3) el adecuado uso del estilo de liderazgo resonante armonizará la articulación del talento humano con la estrategia del negocio, y su adaptación a los cambios del entorno; (4) se hace necesario que indiferentemente del género, el ser humano se dé la oportunidad de apropiar un liderazgo resonante, armonizando su entorno con un enfoque sustentable ante las dinámicas inestable de los mercados, que sea un portador de emociones positivas ante su gente. 
REVISTA DE LA UNIVERSIDAD DEL ZULIA. 3ㄹe época. Año $12 \mathrm{~N}^{\circ}$ 34, 2021

Ricardo. R. Antequera Amaris et al. /// Liderazgo resonante según el género... 29-44

DOI: http://dx.doi.org/10.46925//rdluz.34.03

\section{Referencias}

Antequera Amaris, R. R. y Ospino Acuña, O. A. (2020). Liderazgo resonante en las Pymes panificadoras de la ciudad de Barranquilla. [Tesis de pregrado, Universidad de la Costa]. Repositorio Universidad de la Costa - REDICUC. https://hdl.handle.net/11323/7149

Arteaga, A. y Ramón, S. (2009). Liderazgo Resonante según género. Revista Multiciencias, 9(3), 289-295.

Bavaresco de Prieto, A. M. (2008). Proceso Metodológico en la Investigación. (Cómo hacer un diseño de investigación) (6. eㅡ. $)$. Imprenta Internacional.

Brzovic, P. (2010, 06 de julio). Reportaje sobre Liderazgo masculino y femenino: ies posible complementarlos? MBA América Economía. Disponible en: https://bit.ly/2CAi7K5

Buitrago, R. (2014). El poder, las mujeres y su liderazgo ejercido en el sector público. Revista Fórum Humanes, 3(2), 45-59.

Carocca, H. (2016). Diferencias en el estilo de liderazgo según sexo, de acuerdo a la percepción de Directivos Públicos del II Nivel Jerárquico de la Región de Valparaíso. [Tesis de maestría, Pontificia Universidad Católica de Valparaíso]. Repositorio Institucional PUCV. http://opac.pucv.cl/pucv_txt/txt-8000/UCD8122_0l.pdf

Chávez, N. (2012). Introducción a la investigación educativa. Ars Gráfica Editores.

Cruz, C., Olivares, S. y González, M. (2014). Metodología de la Investigación. Grupo Editorial Patria.

Dankhe, G. (1986). Investigación y comunicación, en C. Fernández-Collado y G.L. Dankhe (eds). La comunicación humana ciencia social. Mc.Graw-Hill, México.

Eagly, A. H., Johannesen-Schmidt, M. C., \& van Engen, M. L. (2003). Transformational, transactional, and laissez-faire leadership styles: a meta-analysis comparing women and men. Psychological bulletin, 129(4), 569-591. https://doi.org/10.1037/0033-2909.129.4.569

Fernández López, G., Rodríguez Palomino, V., Ramírez Molina, R., y Villalobos Antúnez, J. (2020). Enfoques emergentes del liderazgo en las Pequeñas y Medianas Empresas de la ciudad de Barranquilla. Revista Interdisciplinaria De Investigación, 4(4), 23 - 40.

Fritz, C. y Van Knippenberg, D. (2017). Gender and leadership aspiration: the impact of organizational identification. Leadership \& Organization Development Journal, 38(8), 1018-1037.

Goleman, D., Boyatzis, R. y McKee, A. (2016). El Líder Resonante Crea Más: El Poder De La Inteligencia Emocional. Penguin Random House. 
Guerrero Cuentas, H.R., Ramírez Molina, R.I., Herrera Tapias, B., Herrera Mendoza, K., y Avendaño Villa, I. (2018). El ejercicio investigativo Una aplicación desde el quehacer docente de maestros y maestras del Departamento del Magdalena TOMO II. (1.르 ed.). 001-118. Editorial Universitaria de la Costa. Barranquilla, Colombia.

Hernández, R., Fernández, C. y Baptista, P. (2014). Metodología de la Investigación. Editorial McGraw-Hill.

Herrera Tapias, B., Guerrero Cuentas, H. y Ramírez Molina, R. I. (2018). Investigación como estrategia pedagógica: Una mirada desde la educación, escuela y transformación de la comunidad global. Editorial Universitaria de la Costa.

Hughes, D. J., Lee, A., Wei Tian, E., Newman, A. y Alison Legood. (2018). Leadership, creativity, and innovation: A critical review and practical recommendations. The Leadership Quarterly, 29(5), 549-569. https://doi.org/10.1016/j.leaqua.2018.03.001

Hughes, R., Ginnett, R. y Curpy, G. (2007). Liderazgo. Editorial McGraw-Hill.

Hurtado, J. (2010). Metodología de la Investigación. Guía para una comprensión holística de la ciencia. Ediciones Quirón.

Instituto Colombiano de Bienestar Familiar. (2017). Censo de plantas productoras de alimentos regional Atlántico. Disponible en: https://bit.ly/3let9lL

Lanzoni, Ilse. (2008). Perspectiva de género en la reforma del sector público de Costa Rica: Desarrollando mujeres líderes como agentes de cambio. Revista de Servicio Civil de Costa Rica, 1, 43 50.

Magnusson, D. (1978). Teoría de los Test. Editorial Trillas.

Malhotra, N. K. (1997). Investigación de Mercados. Un Enfoque Práctico (2.ํed.). Prentice-Hall. Marañón, F., Barrientos, R. \& Frías, L. G. (2021). Imagen, confianza y liderazgo en voceros públicos durante la pandemia COVID-19. caso: Nuevo León, México. Journal of the Academy, (5), 7-26. https://doi.org/10.47058/joa5.2

Maxwell, J. C. (2007). Las 21 leyes irrefutables del liderazgo (1. 르 ed.). Grupo Nelson Inc.

Ministerio de Comercio, Industria y Turismo. (2019, 5 de junio). Decreto 957. Disponible en: https://bit.ly/2VkGQIB

Miranda, S. R. (2019). Preferred leadership styles by gender. Journal of Management Development, $38(7), 604-615$.

Niño Rojas, V. (2011). Metodología de la Investigación diseño y ejecución. Ediciones de la U. https://bit.ly/3fMloDe 
Ramírez Molina, R. J. (2020). Inteligencia Social y Liderazgo Resonante en instituciones públicas de salud. [Tesis de Maestría, Universidad Privada Dr. "Rafael Belloso Chacín"]. Repositorio Universidad Privada Dr. "Rafael Belloso Chacín". https://www.urbe.edu/portalbiblioteca/basesdedatos-urbe/tesis/

Ramírez Molina, R. J., Marcano, M. V., Ramírez Molina, R. I., Lay Raby, N. D. y Herrera Tapias, B. A. (2019). Relationship Between social intelligence and resonant leadership in public health Institutions. Revista Opción, 35(90), 1223-1249.

Ramírez Molina, Reynier Israel., Chacón Zúñiga, H.C., \& El Kadi Janbeih, O. N. (2018). Gestión estratégica del talento humano en las PYMES. (1. e․ ed.). 001-120. Editorial Corporación CIMTED. Medellín, Colombia.

Ramírez Molina, Reynier Israel; Fernández López, Giselle Paola y El Kadi Janbeih, Omar Nabih. (2021). Estilos de liderazgo predominantes en las pequeñas y mediana empresas. Revista Global Negotium, 4(1), 61-85.

Ramírez Molina, R. J., Ramírez Molina, R. I., Villalobos Antúnez, J.V., Lay Raby, N.D., \& Marcano, M. V. (2021). Inteligencia social y liderazgo resonante. (1. a ed.). 001-129. Editorial Centro Internacional de Marketing Territorial para la Educación y el Desarrollo CIMTED. Medellín-Colombia.

Ramos Ruíz, J. L., Polo Otero, J. L. y Pineda Serna, P. (2013). Plan estratégico departamental de ciencia, tecnología e innovación del Atlántico - PEDCTI 2012 - 2022. Gobernación del Atlántico, Colciencias, BID, Universidad del Norte y Universidad del Rosario. Disponible en: https://bit.ly/3dDcirU

Robbins, S. P. (2004). Comportamiento organizacional. Pearson Educación.

Ruiz Bolívar, C. (1992). La educación en el contexto del desarrollo de América Latina y el Caribe. Memorias del Congreso Hispanoamericano de Investigación Educativa: Encuentro de Dos Mundos por la Paz y el Desarrollo.

Sectorial (2016). Informe sector Industria Panificadora. Informes Sectorial.

Smith, L.M, Andrusyszyn, M.A., \& Laschinger, H.K.S. (2010). Effects of workplace incivility and empowerment on newly-graduated nurses' organizational commitment. Journal of Nursing Management, 18, 1004-1015.

Sukier, H., Ramírez Molina, R. I., y Parra, M. (2020). Modelo de gestión estratégica de talento humano desde un enfoque sustentable. (1. e ed.). 001-101. Editorial Universitaria de la Costa.

Tamayo y Tamayo, M. (2007). Técnicas de la Investigación. Editorial Panapo. 